\title{
Dark Matter as Past Matter, a Conjecture
}

\section{Pierpaolo Perrucci}

Collepasso (LE), Italy

Email: pierpaoloperrucci4@gmail.com

How to cite this paper: Perrucci, P. (2021) Dark Matter as Past Matter, a Conjecture. Journal of High Energy Physics, Gravitation and Cosmology, 7, 452-473. https://doi.org/10.4236/jhepgc.2021.72026

Received: October 2, 2020

Accepted: April 3, 2021

Published: April 6, 2021

Copyright (c) 2021 by author(s) and Scientific Research Publishing Inc. This work is licensed under the Creative Commons Attribution International License (CC BY 4.0).

http://creativecommons.org/licenses/by/4.0/

\begin{abstract}
Up to now several cosmological models have not given yet a satisfactory frame for the existence of Dark Matter (DM) and Dark Energy (DE). This paper attempts to give a geometrical framework for both their emergence and existence. The universe mass/energy densities proportions for Baryonic Matter (BM) and DE exhibit the same relations between the radius and the area of a disc, in which DM matches to the radius squared. That disc may correspond to the base of the Minkowski light cone, which is the front light wave we intercept from the past. I suggest taking time dimension more into consideration, and exploring a new direction on the research of DM and DE as typical relic of BM, positive mass made negative through a continuous phase transition, from a three-dimensional space dimension to the one-dimensional time.
\end{abstract}

\section{Keywords}

Dark Matter, Gravity

\section{Introduction}

In order to justify such a turn-around in the conception of DM, this paper will start with a brief history of DM discovery, and of the many efforts which have been, are and will be deployed for a better understanding. The second paragraph will take into account what the research, together with the observation tools, has produced in matter of DM distribution. In the third we take a quick look to the search and the results for its eventual particle. Then it is taken into consideration an old cosmological conjecture, whose side-effects support the theory presented in the discussion. In the data section it is presented a list of the main assumptions behind the theory, corroborated by the available data. The discussion will demonstrate how a particular geometrical relation, within the Minkowski light cone, matches the proportions observed for BM, DM and DE mass/energy densities. 


\section{Dark Matter (DM)}

\subsection{The Discovery and the Research}

In 1904 Lord Kelvin applied the theory of gazes to the Milky Way, [1] supposing that stars behave like gas particles, requiring major mass density than what is visible, in order not to run away due to galactic centrifugal force. Later in 1906 Poincaré called it generally "matière obscure" [2]. Also Kapteyn [3] in 1922 mentioned DM in his observation of our galaxy. Later his student Oort [4] in 1932 published deeper studies about the DM, whose gravitational pull would may account for the excessive speed of globular clusters around the Milky Way. One year later Zwicky gave more consistency to DM (“... dunkle Materie in sehr viel größerer Dichte vorhanden ist als leuchtende Materie.”), [5] through the observation of the excessive rotational velocity of luminous matter in the Coma galaxy, compared to the calculated mass density of the cluster. The stronger gravitational attraction would be justified only by adding invisible mass to the visible matter density of the cluster. In 1970 Rubin and Ford proved its necessity through their observations of Andromeda galaxy rotation, [6] and in an article published in 1978, [7] they extended their precise measurements on the light-mass ratio (LMR) of ten high luminosity galaxies. Decades later many observations are still based on the same principle, thanks to a much more advanced technology that produced, i.e. the Radial Acceleration Relation (RAR) [8]. A more solid basis for DM existence has been also given by the study of the Microwave Cosmological Background (CMB), [9] which is the thermal picture of the instant in which the first radiation had started to travel freely towards us. By the use of statistics obtained through the multi pole power spectrum and applying spherical harmonics to the multi pole moments, CMB temperature anisotropies revealed and confirmed (besides the black body temperature in the monopole, the solar system relative motion and speed in the dipole) sound waves of matter density fluctuations, which matched various standard model predictions (universe flatness, inflation and baryonic acoustic oscillation among others). It also provided with an estimation of total mass/energy density of matter being roughly one fourth of total universe energy density, of which a slight $5 \%$ baryonic, being the rest called DE [10] [11]. Once DM existence had been widely accepted by the scientific world, astrophysicists needed to know how it is distributed in the universe and what DM is made of. Highly performing tools, observational methods, along with a plentiful of theoretical studies and conjectures (this is only the umpteenth) have been devoted to the issue of the mysterious missing mass, also defined as the leading paradigm to describe the structure and evolution of the Universe at all scales [12]. Just to give an idea of how "hot" is the issue, here is an indicative list over some ongoing and planned experiments concerning DM to the date of this draft: acronyms of direct and indirect dark matter searches ADMX, AMS, AMANDA, ANAIS, ANTARES, ArDM, ATIC, BPRS, CANGAROO, CAST, CDEX, CDMS, Chandra, CLEAN, CoGeNT, COUPP, COSME, CRESST, CUORE, CYGNUS, DAMA/NAI, DAMA/LIBRA, 
DAMIC, DarkSide, DEAP, DM-Ice, DMTPC, DRIFT, EDELWEISS, EGRET, ELEGANTS, FERMI-LAT, GENIUS, GERDA, GEDEON, GLAST, HEAT, HESS, HDMS, ICECUBE, IGEX, INTEGRAL, KIMS, LEP, LHC, LUX, MAGIC, MALBEK, MIMAC, miniCLEAN, NaIAD, NEWAGE, ORPHEUS, PAMELA, Panda-X, PICASSO, PICO, ROSEBUD, SIMPLE, SUPER-K, Suzaku, TEXONO, UKDMC, VERITAS, WArP, Whipple, XENON10/ 100, XMASS, XMM-Newton, ZEPLIN. Upcoming. ADMX-Gen2, ArDM-1t, CTA, DARWIN, EURECA, FUNK, GEODM, HAWC, LSST, LZ, MAJORANA, SuperCDMS, DEAP-3600, $X E N O N 1 T$ [13]. Each of them gathered important governmental economic efforts and innumerable scientific institutions resources (both human and financial) from all over the world.

\subsection{DM distribution}

Up to now no interacting DM particle has been found, not even the graviton, [14] [15] the particle predicted by the standard model mediating the gravitational interaction. After ruling out so many DM candidates, whose evidence would have revealed DM and consequently also its quantitative and qualitative distribution in the universe around us, the gravitational effects remain the only fruitful way to get DM somehow exposed to our eyes. Gravitational lensing represents the main mean of indirect observation of DM. This is why during the last decades some scientific resources have focused on higher standard telescopes (from ground to space, from optical to radio) in order to locate gravity lenses, in the challenging attempt to trace and map DM. In 1979 The Twin Quasar was the first strong lensing observed from the Kitt Peak National Observatory 2.1 meter telescope. Since the 80's the Optical Gravitational Lensing Experiment (OGLE) continued the job until Cosmic Lens All Sky Survey (CLASS), then the Planck and Hubble (HST) space telescopes took over, while newer and more powerful are on the way, as the Giant Magellan Telescope under construction in Chile and expected to be ready in 2029.

There are three main classes of lensing:

- strong lensing: where there are easily visible distortions (Einstein rings, arcs, multiple images);

- weak lensing: statistical coherent distortions of only a few percent (galaxy surveys, $\mathrm{CMB}$ );

- microlensing: the amount of light (or other electromagnetic signal) received from a background object changes in time.

The flat curve of rotational velocity of galaxies and other data showed consistency with a DM halo hosting each galaxy. Since DM became evident at the galaxies scale, an important part of DM studies focus on a better knowledge of galaxy formation and evolution. Although it is a very active area of research in astrophysics, it is not yet free from uncertainties and open questions.

\subsection{What Is DM Made of?}

Since the 80 's the hypotheses about what DM is made of have developed grossly 
on the following subjects: MACHOs [16] (Massive Compact Halo Objects) made either of black holes or neutron stars or white or brown dwarfs; WIMPs [17] (Weakly Interactive Massive Particles) or GIMPs [18] (Gravity Interactive Massive Particles) as neutrinos, axions, supersymmetric particles; and MOND (MOdified Newton Dynamics) theories [19] [20]. Which of them have been able to explain DM nature in the best way?

MOND theories propose an alternative in order to explain why galaxies do not appear to obey current laws of physics. They have not succeeded to get rid of the need of DM completely, failing their main target. Even recently, Nieuwenhuizen (2016) has pointed out that $M O N D, M O G$, Emergent Gravity and $f(R)$ theories avoid dark matter by assuming a change in General Relativity and/ or in Newton's law ... these alternative theories probably can do without Oort (galactic) DM, they need still normal Zwicky (cluster) DM. We have confirmed for $M O N D$, shown for Emergent Gravity and coined for MOG and $f(R)$, that neutrinos can do the job ... if sterile andlor active neutrinos have eV-scale masses. To date MOND, along with its varieties AQUAL, [21] QUMOND [22] and TeVeS [23] Tensor-Vector-Scalar gravity theories, have not shown full consistency with observations, [24] failing in ruling out DM.

At the turn of the $21^{\text {st }}$ century the MACHO, [25] EROS [26] (Experience pour la Recherche d'Objets Sombres) and OGLE [27] (Optical Gravitational Lensing Experiment) Collaborations helped, through gravitational microlensing, to constrain MACHOs contribution to the Milky Way and M31 halos for a less than significative fraction of DM, [28] as for instance primordial black-holes contribute to no more than $0.1 \%$ [29].

The search of wimps, particles interacting with matter only through a weaker force than the weak nuclear one, or just via gravity, has gone two-ways towards both eventual direct and indirect detection. Direct detection could take place via the production of DM particles in accelerators or via the detection of their annihilation and scattering processes. The wimps mostly searched by accelerators are neutralinos, gravitinos, sneutrinos, axinos. They are the partner particles predicted by supersymmetric theories (SUSY) [30] which, if existing, appear now to be out of the energetic range of today's accelerators.

The indirect detection focuses on the hypothetical interaction between WIMPs and ordinary matter on earth, from a modulated recoil of atoms nuclei or electrons, during the travel of our solar system and earth through the DM halo; and on an hypothetical energy transfer, triggering signals such as scintillation light, phonon signals, ionization signals, bubble generation. Detectors such as directional, inorganic crystal, cryogenic, noble liquid and bubble chambers have been built for this purpose. However Schumann (2019) concludes no experiment observed a statistically significant excess above its background expectation. As of today, the WIMP still remains elusive [31]. In the meanwhile the search for gamma rays, neutrinos and charged cosmic rays, whose behaviour can be explained as WIMP annihilation or decay to yield standard model particles in dense regions of the Universe, is still on progress, but to date it has produced no 
more than constraints and bounds in several directions [32].

More valid candidates for DM have neither been found by Very Energetic Radiation Imaging Telescope Array System (VERITAS), IceCube Neutrino Observatory, Super-Kamiokande Neutrino observatory, Fermi-LAT space telescope, GAIA satellite; nor by the on-going experiments on scattering with nuclei by the Cryogenic Dark Matter Search (CDMS), Cryogenic Rare Event Search with Superconducting Thermometers (CRESST), COherent GErmanium Neutrino Technology (COGENT); while Large Underground Xenon experiment (LUX), Dark Matter-Large Sodium Iodide Bulk for Rare processes (DAMA-LIBRA), Sodium Iodide with Active Background Rejection (SABRE) CDMS (Cryogenic Dark Matter Search), Experience pour DEtecter Les Wimps En Site Souterrain (EDELWEISS), Cryogenic Rare Event Search with Superconducting Thermometers (CRESST) and Liquid Xenon Time Projection Chamber (XENON1T) collaborations as the Small Astronomy Satellite (SAS), COS-B, Energetic Gamma Ray Experiment Telescope (EGRET) and the Fermi Gamma-Ray Space Telescope satellite missions have to date not led to $\mathrm{x}$-gamma rays flux produced by DM particles annihilation, concluding that gamma rays observed could reasonably belong to other already known sources. Operating for some decades now, the Axion Dark Matter eXperiment (ADMX) collaboration has not yet observed photons emitted by the eventually coupling of DM with the magnetic field, putting more and more stringent constraints to the hypothetical axion particle. To similar conclusions, but with less strict constraints for DM, have come the CAPRICE, BESS, AMS, AMS-02, PAMELA, AMANDA IceCube and ANTARES experiments about the cosmic-ray antiproton spectrum eventually produced by annihilation of DM. Therefore none of hypothetical sterile neutrinos, heavy neutrinos or sneutrinos have been found to date (Bertone 2018).

In the meantime the robust support of mighty computer simulations and direct observations ruled out (interacting) hot $\mathrm{DM}$ and (weakly interacting) warm $\mathrm{DM}$, in favour of a (non-interacting) cold DM, [33] which best fitted with the formation through a bottom-up sequence of the large-scale structures of observable universe. A recent study described DM halos around dwarf satellite galaxies being at its maximum entropy, [34] that is to say a full stand-still of its particles (cold) and a completed evolution (past).

Thus, despite its darkness, we may have very good reasons [35] for having DM within the Standard Model, whose name $\Lambda$ CDM however entails another mystery: the so-called Dark Energy $\Lambda(\mathrm{DE})$.

\subsection{Large Number Hypothesis (LNH)}

At the beginning of $20^{\text {th }}$ century astronomer Lemaitre published an article describing the relation between the distance and the Doppler effect observed on galaxies by astronomers [36]. Eddington noticed that such relation seemed like the repulsive term in Einstein's field equation [37]. In 1929 one of those astro- 
nomers, Hubble, fixed such relation known today as Hubble Law. [38] The redshift generated by the receding velocity of galaxies has been measured with more accurate precision, ranging today from 0.67 to $0.74 \mathrm{~km} / \mathrm{s} / \mathrm{Mpc}$, depending respectively on CMB or on cosmic distance ladder [39] [40]. The reason for this discrepancy has not yet found satisfactory explanations, [41] apart from an eventually uneven density throughout space between us and the ladder used [42]. All observations confirm such universe expansion and, after a discovery in 1998, also that universe expansion is apparently accelerating [43] [44]. The same year a paper [45] retrieved a sixty years old theory, Dirac's Large Number Hypothesis (LNH), [46] [47] looking for a theoretical confirmation of such a discovery. In 1937 Dirac had noticed how the relating ratios of size scales of universe might correspond to the fundamental force scales. In his view those were not simply coincidences, but consequences of a cosmology based on following features: gravity is inversely proportional to the age of the Universe; the mass of Universe is proportional to the age of the Universe squared; physical constants are not constants, i.e. they scale as with respect to the age of the Universe. Consequently, in order to make this cosmology work, one of the mean side-effect was that two kinds of mass were needed: one additive in intergalactic space and one multiplicative within matter. About the first, in 1974, [48] Dirac wrote: The only way in which we can preserve the Einstein equations, which demand conservation of mass, is to suppose that, together with the $H$ atoms, a uniform distribution of negative mass is created, so as to make the total density of created matter zero. In order not to have violent disagreement with observation we must suppose that the negative mass is not observable and so is not quantized, like the $H$ atoms. It must not interact with other matter, except gravitationally, and must have no physical effects at all, apart from producing a curvature of space. The $H$ atoms condense into nebulae and stars and form the matter that we observe. The negative mass remains uniform and unobservable." And about the second: "Multiplicative creation requires that all forms of matter shall be multiplying, with the number of atoms increasing proportional to $t^{2}$. It is a little difficult to understand how this can take place in the case of a crystal. Presumably the new atoms must appear on the outside. Hard not to recognize in those lines what we would call DM and DE. Another paper focusing on LRH noticed that "The acceleration of the present universe is a proof of the decrease of the gravitational constant $G$, because the decrease of $G$ corresponds to an effective repulsion", [49] not agreeing with Dirac on the existence of negative mass, which instead explain both DE and DM emergence. Even General Relativity did not exclude negative energy particles, i.e. negative mass, [50] in its description of gravity and laws of motion [51]. Another study about LNH, in 1998, had used the coincidences in order to define to define the value for the energy of the vacuum in quantum field theory, released at the epoch of GUT phase transition, driving primordial universe dynamics according to the inflation theory. Instead of a gravity varying with time, it has then produced a scaling law of the cosmological constant. 
A further paper on LNH described it as a consequence of the holographic principle, provided that the universe can be approximately described by a spatially homogenous, isotropic, and flat cosmological model and that the main contribution to the present energy density comes from the cosmological constant, it is possible to explain all the scaling relations that motivated Dirac's large number hypothesis appealing exclusively to basic principles and to the $N$ bound conjecture [52]. Apart from the present paper, unmistakably LNH continues to stimulate the scientific debate [53] [54].

\subsection{About Time}

Time share some aspects with DM. It never shows signs of instability, nor it is tangible. Despite huge scientific developments in the knowledge of the universe time still remains matter of discussion [55] [56]. Following Minkowski's description, it is as another dimension, [57] like space, in the four-dimensional universe of our current cosmological $\Lambda \mathrm{CDM}$ [58] [59] [60]. Its dimension crosses our three-dimensional space transversally, as a dimensionless point. Gravity interacts with matter through the centre of mass, which is also a dimensionless point. In this way mass puts in relation the time and the space dimensions. Without mass/energy there is no way of measuring time. Time is measurable, the question is if it is also physically existing. There is no apparent reason for not theorizing whether time dimension too, like space, may harbour mass. If so, then it must be one-dimensional and of the opposite sign with respect to the ordinary mass, lying totally to the negative side of the light cone, since evidently, we have no idea if a future time already exists. Spatial mass is positive, while temporal mass (from now on called past mass) must be negative. How to find evidence for such mass? Time is one-dimensional. It is measurable through the comparison of steady physical statuses from different moments. Any point in space, at every instant, i.e. each hyperspace-layer, is timeless. The peculiarity of time dimension, which makes it so different from the three space ones, is that we can measure no matter how far in past direction, while not at all in the opposite (future). The maximum velocity limit imposed in nature by the speed of light $c$ makes anything slower than $c$, casually connected with the observer. Anything faster than $c$ is out, in all terms. Our present, our worldline, travels towards the future and radiates all around at $c$ speed. The same happens with the present of other events. We can see the present of a phenomenon only when its lightcone cross ours. That is to say when its light cone lateral surface crosses ours. We cannot literally watch outside such intersection, before or after that moment, slower or faster than $c$. Inside our lightcone facts are casually connected with us, it is the past. Those happen outside not. Light waves from the past reach us, one after another. Each observation is forced to catch one instant per time. The past might be there although, confined in the bulk, between the origin and the vertex of our lightcone, from the top of which we watch back (temporally back, though spatially around). Our line-of-sight is forced onto the two-dimensional 
flat lateral surface of the light cone. At every instant a spherical lightwave meets our observations, carrying all Universe's four-dimensional information on the two-dimensional image we intercept.

\section{Ground Assumptions and Data}

Despite no experimental data has been produced with the innovating idea of DM as past mass, there are still good reasons in its favour, when following those assumptions:

1) Universe is made of three indistinguishable space dimensions and one time dimension (time is one-dimensional);

2) Light travels at constant speed on the lateral surface of the observer's light-cone;

3) DM interacts with ordinary matter through gravitational interaction only;

4) DM has been found around galaxies, satellite galaxies and cluster of galaxies, for the most;

5) $\Lambda \mathrm{CDM}$ model mass/energy density observed for BM, DM and DE may scale $72.2 \%, 22.9 \%$ and $4.7 \%$;

6) DM and time show strict correlation.

Here follow the studies and the data on which above statements have been based:

1) Universe is made of three indistinguishable space dimensions and one time dimension (time is one-dimensional).

Though string theories have suggested the existence of more "hidden" dimensions, the dimensionality on which General Relativity is based, which alone has already given solid predictability, requires the universe dimensions to remain 3 +1 . Tegmark [61] demonstrates in one of his studies, that it is far from obvious that any other $(n, m)$ permits observers, since radical qualitative changes occur in all cases, so that the burden of proof of the contrary falls on the person wishing to criticize ensemble theories with fine-tuning arguments. Any other combination than four dimensions, has fewer probabilities yielding both the stability and the predictability of our universe. In the abstract of his study, he clearly explains With more or less than one time dimension, the partial differential equations of nature would lack the hyperbolicity property that enables observers to make predictions. In a space with more than three dimensions, there can be no traditional atoms and perhaps no stable structures. A space with less than three dimensions allows no gravitational force and may be too simple and barren to contain observers." Then he concludes given the other laws of physics, it is not implausible that only a $(3+1)$-dimensional spacetime can contain observers that are complex and stable enough to be able to understand and predict their world to any extent at all, for the following reasons.

- More or less than one time dimension: insufficient predictability.

- More than three space dimensions. insufficient stability.

- Less than three space dimensions: insufficient complexity. 


\section{2) Light travels on the lateral surface of a light-cone.}

During last hundred years Einstein's special relativity has been tested successfully by innumerable experiments giving to Minkowski light-cone an experimental validity. Distances between two points, in a four-coordinate system as spacetime, are named intervals. As Feynman explains if we consider a point which in a given coordinate system had zero time, and only space, then the interval squared would be negative and we would have an imaginary interval ... Intervals can be either real or imaginary in the theory ... When an interval is imaginary, we say that the two points have a space-like interval between them (instead of imaginary), because the interval is more like space than like time. On the other hand, if two objects are at the same place in a given coordinate system, but differ only in time, then the square of the time is positive and the distances are zero and the interval squared is positive; this is called a time-like interval. Therefore at $45^{\circ}$ there are two lines (actually, in four dimensions these will be cones, called light cones) and points on these lines are all at zero interval from the origin. Where light goes from a given point is always separated from it by a zero interval. ... If light travels with speed $c$ in one system, it travels with speed $c$ in another, for if the interval is the same in both systems, i.e., zero in one and zero in the other, then to state that the propagation speed of light is invariant, is the same as saying that the interval is zero [62]. Thus our line-of-sight lies on the lateral surface of the hypercone.

3) DM interacts with ordinary matter through gravitational interaction only.

The discovery of invisible DM has been made by watching the visible matter: through anomalies in the rotational speed of spiral galaxies; in the confinement of hot gas in galaxies and clusters of galaxies; in the apparent random motions of galaxies in clusters; through the gravitational lensing of background objects; and in the observed fluctuations in the cosmic microwave background radiation. Those observations have all been explained by the existence of additional invisible mass [63]. One of the most spectacular proof of such discovery has been made observing the so-called Bullet Cluster, or galaxy cluster (GC) 1E0657-56 [64]. Here DM along with the cluster distort the space gravitationally, well aside from the baryonic gas, containing the most of ordinary matter. With the help of Chandra satellite two large clouds of hot gas, called the Intra Cluster Medium (ICM), have been detected in $\mathrm{x}$-rays fairly separated from most of the mass of the two fast collided galaxy clusters, inferred by the gravitational lensing observed by the Hubble Space, ESO and Magellan telescopes. The composite picture shows most of the gravitational mass being at the outer ends, while most of baryonic mass remains closer to the centre of collision. It has been interpreted as if most of the baryonic Figure 1, or ordinary matter, contained in the pink clouds is slowed by the drag force induced by the collision, while the blue areas show the clusters along with DM, travelling freely away from each other. There is also a second case, MACS J0025.4-1222 Figure 2, which shows the same behaviour and many others will certainly follow. 


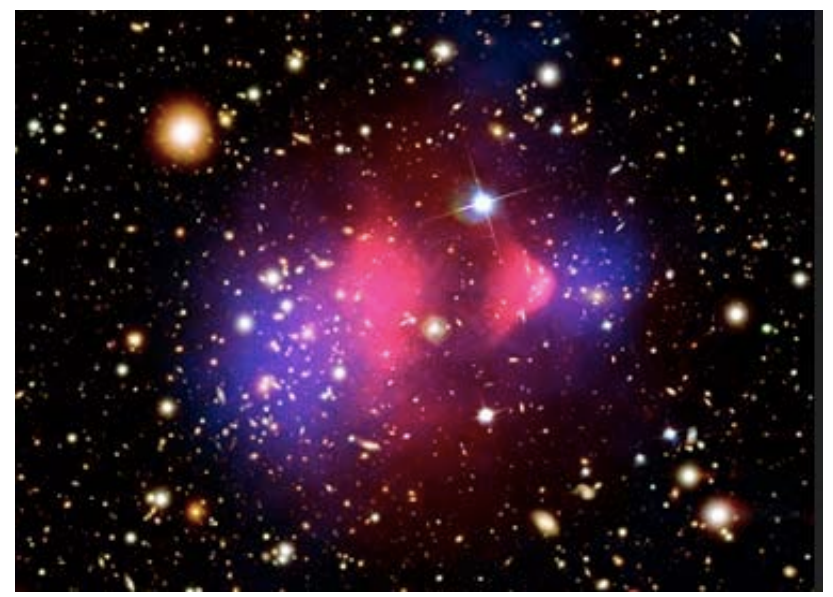

Figure 1. The bullet cluster. The matter in galaxy cluster 1E0657-56, fondly known as the "bullet cluster", is shown in this composite image. A mere 3.4 billion light-years away, the bullet cluster's individual galaxies are seen in the optical image data, but their total mass adds up to far less than the mass of the cluster's two clouds of hot x-ray emitting gas shown in red. Representing even more mass than the optical galaxies and $\mathrm{x}$-ray gas combined, the blue hues show the distribution of dark matter in the cluster. Otherwise invisible to telescopic views, the dark matter was mapped by observations of gravitational lensing of background galaxies. In a text book example of a shock front, the bullet-shaped cloud of gas at the right was distorted during the titanic collision between two galaxy clusters that created the larger bullet cluster itself. But the dark matter present has not interacted with the cluster gas except by gravity. The clear separation of dark matter and gas clouds is considered direct evidence that dark matter exists. Text and Composite Credit. $X$-ray: NASA/CXC/CA/M. Markevitch et al.; Lensing Map: NASA/STSCI; ESO WFI; Magellan/ U. Arizonal D. Clowe et al.; Optical: NASA/STSCI; Magellan/U. Arizonal D. Clowe et al.

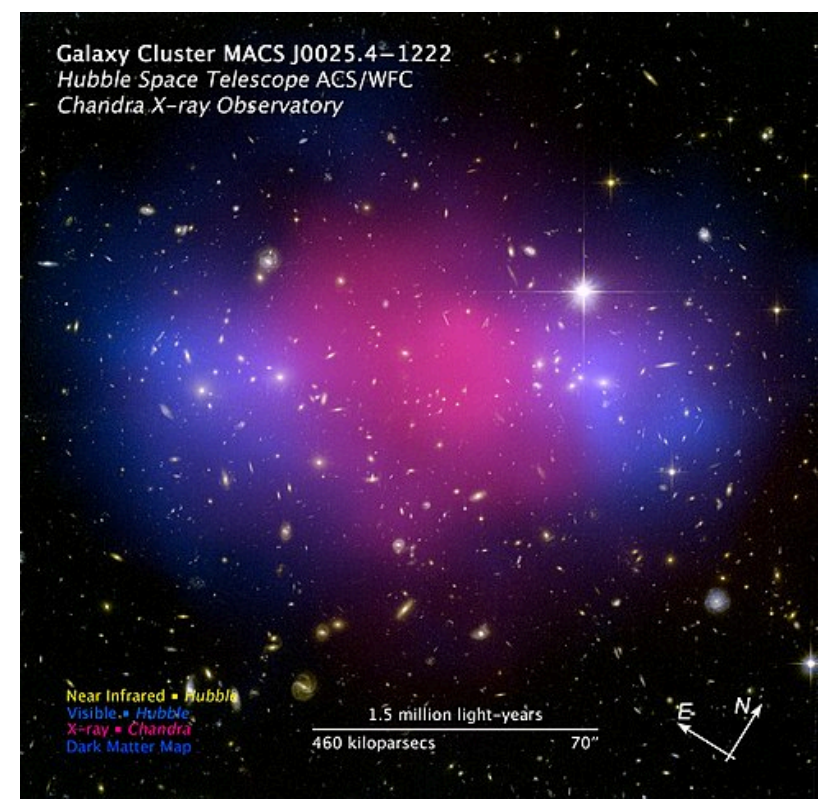

Figure 2. The galaxy cluster MACS J0025.4-1222. Captured by Chandra and Hubble Space Telescope. The intracluster medium, the most of ordinary matter, lies in the middle, whilst the most of mass is far apart. Credits. NASA, ESA, CXC, M. Bradac (University of California, Santa Barbara), and S. Allen (Stanford University) http://hubblesite.org/newscenter/archive/releases/2008/32/. 
4) DM has been found around galaxies, satellite galaxies and cluster of galaxies, for the most.

Recent mappings of DM have become spectacularly wide and rich. Both thanks to the computer simulations, which, it must be underlined, to-day have just given an idea of how DM real distribution might be, and to the enhanced technology deployed in telescopes. The main search for DM peculiar properties has focused on galaxy formation computer modelling; measuring galaxy abundances, galaxy clustering as their clustering properties (mass, redshift and scale); listing group and cluster catalogues; comparing galaxies star formation rate (SFR), the halo occupation distribution (HOD), the galaxy-halo connection, the Stellar-to-Mass Halo Ratio (SMHR), the radial distribution of matter or substructures within halos and the velocity distribution of DM or of substructures within halos. Both empirical and physical models have been created in order to fix a galaxy-DM halo connection and the interaction in between. While lensing directly deduces the mass of DM halos around galaxies, in a clustering analysis instead the masses are inferred indirectly from the relationship between the halo mass and the clustering. Datasets showed that baryonic matter follows the distribution of dark matter even on large scales [65]. Data on DM are consistent with a model in which $\operatorname{og} M=0.2$ or somewhat smaller, which is consistent with constraints from clustering, lensing, and group statistics [66].

5) $\Lambda \mathrm{CDM}$ model mass/energy density observed for BM, DM and DE may scale $72.2 \%, 22.9 \%$ and $4.7 \%$

The $\Lambda \mathrm{CDM}$ model predictions of its cosmological parameters have been researched and constrained by studying the microwave radiation of CMB. COBE [67] satellite (Cosmic Background Explorer) has observed that CMB has a near perfect black-body spectrum and the existence of very faint anisotropies. The main instruments on-board, the DMR (Differential Microwave Radiometers), [68] have mapped the variations (anisotropies) in the CMB, determining the CMB dipole amplitude and direction; while the Far-InfraRed Absolute Spectrophotometer (FIRAS) [69] has measured the spectrum of the CMB. With the help of the Wilkinson Microwave Anisotropy Probe satellite (WMAP) [70], temperature and polarization sky maps are examined to separate CMB anisotropies from foreground emission, and both types of signals have been analysed in detail [71] providing with more refined and accurate measurements and constraints to cosmological parameters, such as the density of atoms; the density of all other non-atomic matter; the epoch when the first stars started to shine; the "lumpiness" of the universe, and how that "lumpiness" depends on scale size. Most recent datasets have been released with the help of Planck [72] satellite (initially called COBRAS/SAMBA: the Cosmic Background Radiation Anisotropy Satellite/Satellite for Measurement of Background Anisotropies). Its datasets [11] [73] show that the Planck spectra at high multipoles are extremely well described by the standard spatially-flat six-parameter $\Lambda C D M$ cosmology with a power-law spectrum of adiabatic scalar perturbations. Within the context of this cosmology, the Planck data determine the cosmological parameters to high precision. 
Planck was the first submillimetre mission to map the entire sky to sub-Jansky sensitivity (a non-SI unit for spectral flux density: $1 \mathrm{Jy}=10-26 \mathrm{~W} \cdot \mathrm{m}^{-2} \cdot \mathrm{Hz}^{-1}$ ) [74] with angular resolution better than 10'. Observations show that the CMB contains anisotropies over a wide range of angular scales. These anisotropies are usually expressed by using a spherical harmonic expansion of the CMB sky. Another fundamental source of information on cosmological parameters is the $\mathrm{BAO}$ (Baryonic Acoustic Oscillation) as the perturbations of the proton-electron plasma coupled to photons subject to gravitational instability. The fluid density underwent oscillations, giving time variations in temperature. These combine with a velocity effect, which is $\pi / 2$ out of phase and has its amplitude reduced by the sound speed. After the Universe recombined, the radiation decoupled from the baryons and could travel freely towards us. That is the image of CMB's harmonic series of peaks. The physical length scale associated with the peaks is the sound horizon at last scattering. This length is projected onto the sky, leading to an angular scale that depends on the geometry of space, as well as the distance to last scattering. Hence the angular position of the peaks is a sensitive probe of a particular combination of cosmological parameters. In fact, the angular scale, is the most precisely measured observable, and hence is usually treated as an element of the cosmological parameter set ... One can think of them as a snapshot of stochastic standing waves. Since the physics governing them is simple and their structure rich, one can see how they encode extractable information about the cosmological parameters [75]. Those figures are based on the strong assumptions of the $\Lambda \mathrm{CDM}$ model and consequently extrapolated from the data collected. Despite the enormous success of the mission bringing very strong concordance with the predictions and the most advanced technology used, still there exist some uncertainties among what registered by BOOMERANG, DASI + DMR, WMAP and PLANCK projects [76]. One for all the mismatch of the Hubble parameter between the value of $67.4 \mathrm{~km} / \mathrm{sec} / \mathrm{Mpc}$, in significant disagreement with the rate of $74.0 \mathrm{~km} / \mathrm{sec} / \mathrm{Mpc}$ measured with Cepheid stars [77]. The Hubble parameter constant mismatch, for instance, may require an update of the cosmological model behind the measurements. Furthermore different estimations have been made depending on the priors and ladders used [78]. The actual calculated proportions derived from data observed range grossly from a $68 \%$ to a $73 \%$ of $\mathrm{DE}$, from a $22 \%$ to a $26 \%$ of $\mathrm{DM}$ and from a $4 \%$ to a $5 \%$ to $\mathrm{BM}$ Figure 3 well within the values observed (Figure 4).

\section{6) DM and time show correlation}

It has been noticed a strict correlation between mass of galaxies (and eventually different phases of their evolution) and the DM halos which harbour them [79]. The galaxies and the mass of hosting DM halos run very closely, not in contrast with a simultaneous evolution.

\section{Discussion}

Summarizing DM show following features: it is stable (no decay has been observed), it is cold (non-interacting with strong, nor electroweak forces), it is 


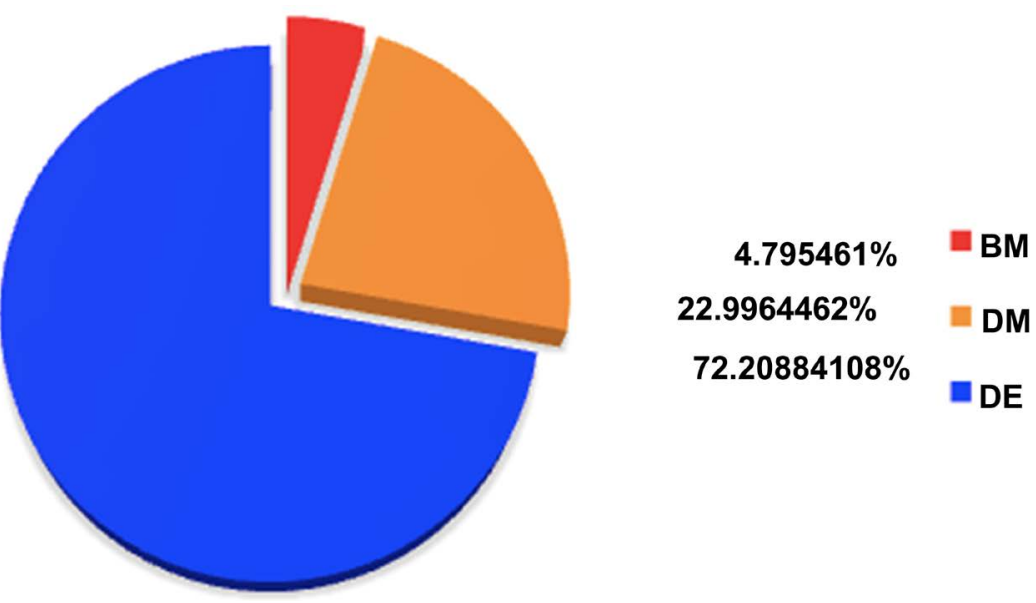

Figure 3. Hypothetical geometrical proportions among BM, DM and DE mass/energy densities. Those values may fit exactly the proportions among BM, DM and DE, for DM $=\mathrm{BM}^{2}$ and $\mathrm{DM}^{*} \pi=\mathrm{DE}$.

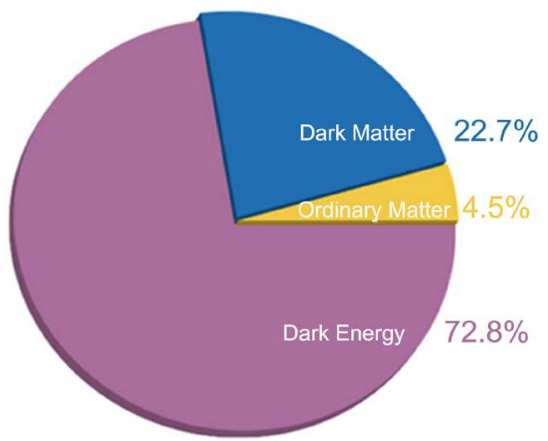

Before Planck

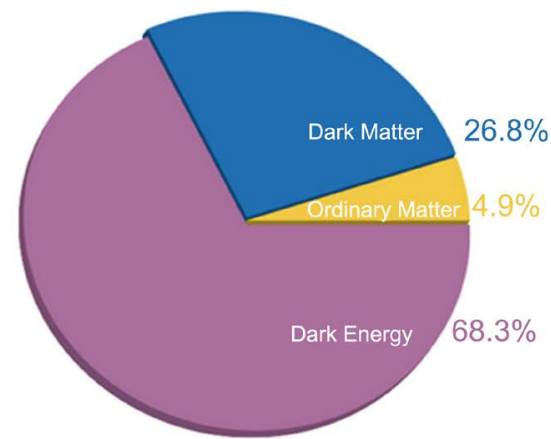

After Planck

Figure 4. Calculated proportions of mass/energy densities of universe from the CMB. There exist still uncertainties about exact proportions among BM, DM and DE for the energy and mass densities observed by WMAP and PLANCK missions respectively. Credit. https://www.esa.int/Science Exploration/Space Science/Planck/Planck reveals an almo s perfect Universe.

time-correlated (with the evolution phase of the hosted galaxy), it is growing (along with star formation or outpacing the galaxy quiescence), it is massive (much more than hosted ordinary matter).

What we actually see, what we normally call observable universe, should be the physical phenomena met by our light cone in one instant of the past, for the limited speed of light cannot reach us instantaneously from anywhere (Olbers' paradox). Thus what we are used to guess as the universe content should be inferred from what our line of sight catches in a given instant, the picture of that moment (or a collection of them).

The present mass, at one end of the arrow of time, must have existed also in the past. If time dimension is still there as the past light cone, then past mass should be there too. Now, while all four dimensions are taken into consideration when calculating universe quantities, observations are necessarily limited to two 
space dimensions only, as two-dimensional is the image of the front lightwave meeting us. The relations among those figures seem to suggest an underlying two-dimensional geometry: within them we can find the values $72.2 \%, 22.9 \%$ and $4.7 \%$. Those proportions correspond to the radius of disc, its radius squared and the area. The same proportions could be applied to the above mentioned light cone on its radius and base. It would be feasible that if at every instant our line of sight catches, along the lateral surface of the light cone, the crossing of such radius, the latter would return a two-dimensional space-like timeless picture of the past. DM cannot be seen nor touched, its presence can be only inferred thanks to the gravitational interaction. If DM lies beyond our line of sight, temporally beyond, not spatially, we would not be able to literally see it. This may be why our entire estimation of the mass/energy density in the universe may belong mostly to past mass/energy. DM and DE may fill the gap between the origin and the present mass/energy according to the equations:

$$
\begin{gathered}
\mathrm{DM}=\mathrm{BM}^{2} . \\
\mathrm{DM}^{*} \pi=\mathrm{DE} .
\end{gathered}
$$

In Figure 5 the radius carries the information of the gravitational weight of mass. An interesting similitude can be found in Newton's Law of Universal Gravitation, where the gravitational force is inversely proportional to the square of the distance between the two masses, distance also named radius, adding support to gravity as coupling time dimension with space. Law of Universal Gravitation however puts in relation two bodies interacting gravitationally. If their centre of masses would trace the crossings of time dimension with space, through which gravity may interact, then the analysis on BM, DM and DE would put our observation in relation with the rest of the universe as a whole.

Negative mass has, probably, [80] never been observed, though mathematically its existence is wholly consistent [81]. In theory, negative and positive mass should behave as follows:

- Positive mass attracts positive mass (gravitation);

- Negative mass repels both positive and negative mass (DE);

- For different signs, there is a push that repels the positive mass from the negative mass, and a pull that attracts the negative mass towards the positive one at the same time. That is to say that positive mass runs away from negative mass, which runs after it (the so-called runaway motion) [50].

Through computer simulations [82] experimental physics has tried to find out how LSS have formed, up to the situation of filaments we observe today Figure 6. They run on the principle that pre-existing DM has gathered matter and then decayed and/or scattered. This paper aims to suggest instead that DM and DE may be born (almost) together with BM. Then the sticky DM and repulsive DE would have respectively attracted and pushed matter together, from the very start until today. Actual expansion of universe may be at the origin of the wider size of DM around BM (halos), given the huge time scale involved. Then DM halos from early universe should appear denser and smaller, e.g. as in a recent 


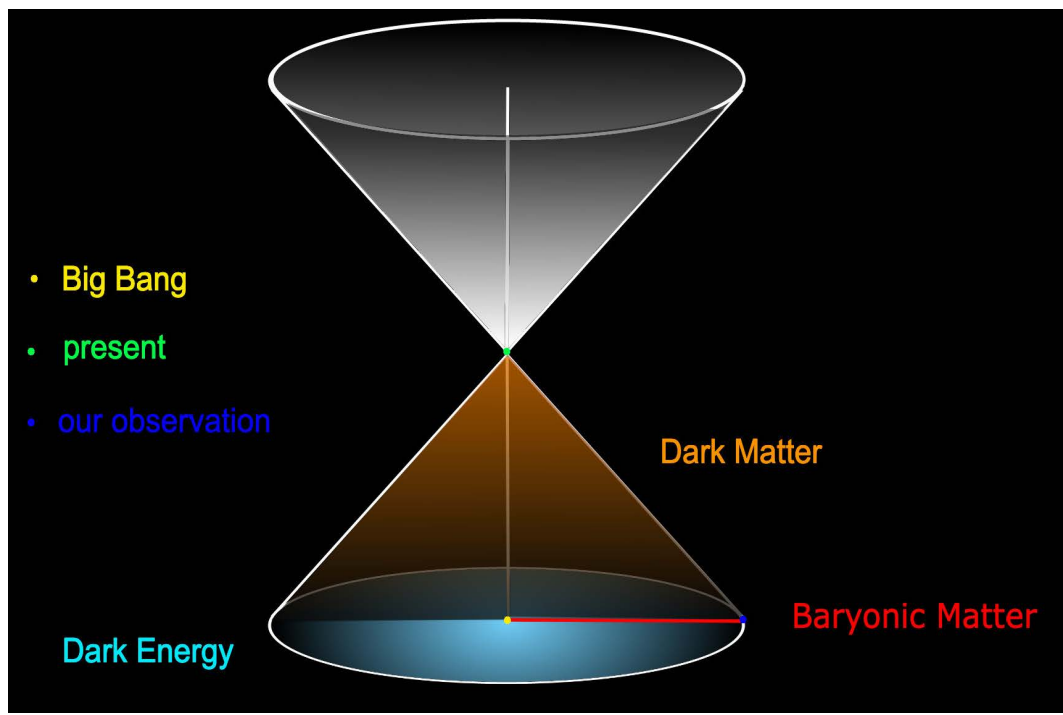

Figure 5. The lightcone geometry behind mass/energy densities of universe from CMB. The graphical representation of mass/energy density information contained in the intersection (blue spot) between our line of sight and light waves. Within the lower nappe of our lightcone the radius is in red, the radius squared in orange, the base area of the cone in light blue; the yellow spot is the Big Bang, the green spot is our present and the blue spot is our object of observation. Note that in this picture one dimension has been left out.

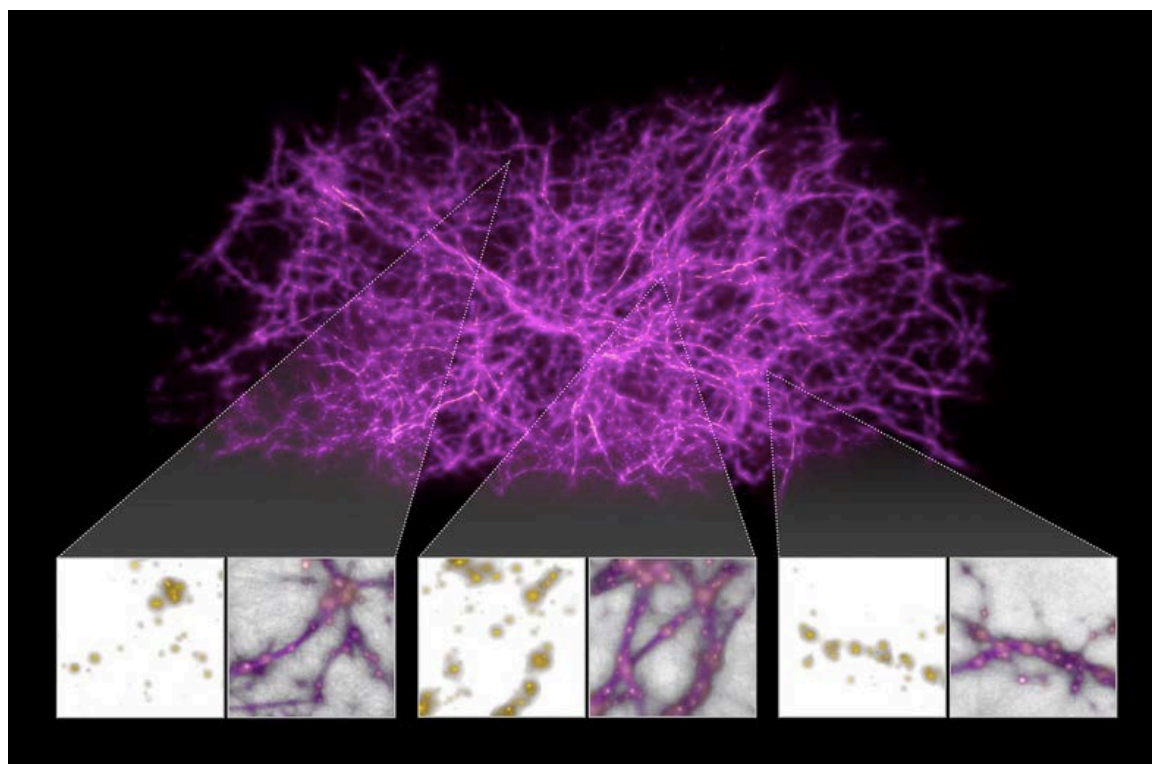

Figure 6. Computer simulation of dark matter filaments in the universe. Astronomers have designed a computer algorithm, inspired by slime mould behaviour, and tested it against a computer simulation of the growth of dark matter filaments in the Universe. The researchers then applied the slime mould algorithm to data containing the locations of over 37,000 galaxies mapped by the Sloan Digital Sky Survey. The algorithm produced a three-dimensional map of the underlying cosmic web structure. They then analysed the light from 350 faraway quasars catalogued in the Hubble Spectroscopic Legacy Archive. These distant cosmic flashlights are the brilliant black-hole-powered cores of active galaxies, whose light shines across space and through the foreground cosmic web. Credit. NASA, ESA, and J. Burchett and O. Elek (UC Santa Cruz)

https.// esahubble.org/ images/ heic2003al 
discovery, which showed a very ancient galaxy harbouring a nut of dense DM [83].

\section{Conclusions}

As already mentioned before, decades of experimental physics have not yet produced evidence of what DM really is, while making it absolutely necessary. The exact geometrical proportions in Equations (1) and (2), the observation on DM behaviour and the lack of to-date satisfying explanation should address the research to take into consideration a change of direction, regardless of how bizarre it may seem, at least to rule it out definitively. The innovation introduced by this paper is theorizing time as a physical existent quantity, measurable through the amount of cumulated DM, observed through the gravitational lenses. It is also inspired by the so-called dark fluid, repelling itself in the big voids, among the filaments of large-scale structures (LSS) and sticking to matter in halos and clumps [81]. Within this frame if the most of the mass and most of the energy of universe really hide themselves within the negative nappe of the light cone, then they should respectively be DM and DE. The above conjectured existence of negative mass would also explain the direction of time arrow, forced by the runaway motion, consequent to the behaviour of mass of opposite signs. It would also be responsible for the growth of universe, both spatially (increasing the volume of the bulk) and temporally (pushing ahead our present on the edge of the light cone).

The research on galaxies and galaxy clusters velocities and direction of motion, their comparison with their DM halos would be able to demonstrate whether this study aims in the right direction. According to this conjecture a young or fast moving galaxy would not be able to gather enough matter to grow, since it would be lacking the important contribution from DM gravitational pull (not cumulating enough mass on a small space or time) showing consequently a thin halo condensation trail or lacking DM at all. Slow and/or old galaxies instead would exhibit wide and dense DM halos.

Despite cosmology of DM remains the main subject of this paper, however it unavoidably raises several questions within the particle physics of the Standard Model. While on cosmic scale it is still reasonable to enhance DM resolution in order to better define its behaviour, on the other hand, on the particles scale, it would be a unique challenge to reveal the existence of a messenger particle. Given the geometrical structure above mentioned, a hypothetical messenger particle of DM would correspond to that of gravitational interaction and be wholly time-like, like a wave, for instance. Some MOND, MOG and $f(R)$ or extended theories of gravity, which share with this paper a time dependent gravitational constant and according to whom DM and DE would be emergent phenomena, do in fact suggest the eventual type of polarization in gravitational waves (GW) would directly tell whether GR has to be overcome by a more complete theory of gravitation. However to-date no "forbidden" polarization of GW has 
been found, nor excluded [84]. For this reason, along with the space observation of DM halos properties, the strong technological improvement, [85] which is globally devoted to the detection and study of GW, will certainly offer new important hints for a fruitful exchange between cosmology and particle physics with undoubted gain for the whole world of science.

\section{Conflicts of Interest}

The author declares no conflicts of interest regarding the publication of this paper.

\section{References}

[1] Kelvin, L. (1902) I. On the Clustering of Gravitational Matter in Any Part of the Universe. Philadelphia Magazine, 3, 1-9.

[2] Poincaré, H. (1906) La voie lactée et la théorie des gas. Bulletin de la Societé Astronomique de France, 20, 153-165.

[3] Kapteyn, J.C. (1922) First Attempt at a Theory of the Arrangement and Motion of the Sidereal System. Astrophysical Journal, 55, 302-327. https://doi.org/10.1086/142670

[4] Oort, J.H. (1932) The Force Exerted by the Stellar System in the Direction Perpendicular to the Galactic Plane and Some Related Problems. Bulletin of the Astronomical Institutes of the Netherlands, 6, 249-287.

[5] Zwicky, F. (1933) Die Rotverschiebung von extragalaktischen Nebeln. Helvetica Physica Acta, 6, 110-127.

[6] Rubin, V. and Kent Ford Jr., W. (1970) Rotation of the Andromeda Nebula from a Spectroscopic Survey of Emission Regions. Astrophysical Journal, 159, 379. https://doi.org/10.1086/150317

[7] Rubin, V., Ford, W.K. and Thonnard, N. (1978) Extended Rotation Curves of High-Luminosity Spiral Galaxies. IV—Systematic Dynamical Properties, Sa through Sc. Astrophysical Journal, 225, L107-L111. https://doi.org/10.1086/182804

[8] Di Paolo, C., Salucci, P. and Fontaine, J.P. (2019) The Radial Acceleration Relation (RAR): The Crucial Cases of Dwarf Discs and of Low Surface Brightness Galaxies. The Astrophysical Journal, 873, Article No. 106. https://doi.org/10.3847/1538-4357/aaffd6

[9] Penzias, A.A. and Wilson, R.W. (1965) A Measurement of Excess Antenna Temperature at $4080 \mathrm{Mc} / \mathrm{s}$. Astrophysical Journal, 142, 419-421. https://doi.org/10.1086/148307

[10] Jarosik, N., Bennett, C.L., Dunkley, J., Gold, B., Greason, M.R., Halpern, M., et al. (2011) Seven-Year Wilkinson Microwave Anisotropy Probe (WMAP) Observations: Sky Maps, Systematic Errors, and Basic Results. The Astrophysical Journal Supplement Series, 192, Article No. 14. https://doi.org/10.1088/0067-0049/192/2/14

[11] Planck Collaboration (2020) Planck 2018 Results: I. Overview and the Cosmological Legacy of Planck. Astronomy \& Astrophysics, 641, Article No. A1. https://doi.org/10.1051/0004-6361/201833880

[12] Bertone, G. and Hooper, D. (2018) A History of Dark Matter. Reviews of Modern Physics, 90, Article No. 45002. https://doi.org/10.1103/RevModPhys.90.045002

[13] Nieuwenhuizen, T.M. (2016) How Zwicky Already Ruled out Modified Gravity 
Theories without Dark Matter. Fortschritte der Physik, 65, Article ID: 1600050. https://doi.org/10.1002/prop.201600050

[14] Rothman, T. and Boughn, S. (2006) Can Gravitons Be Detected? Foundations of Physics, 36, 1801-1825. https://doi.org/10.1007/s10701-006-9081-9

[15] Freeman, D. (2013) Is a Graviton Detectable? International Journal of Modern Physics A, 28, Article ID: 1330041. https://doi.org/10.1142/S0217751X1330041X

[16] Griest, K. (1991) Galactic Microlensing as a Method of Detecting Massive Compact Halo Objects. Astrophysical Journal, 366, 412. https://doi.org/10.1086/169575

[17] Vittorio, N. and Silk, J. (1984) Fine-Scale Anisotropy of the Cosmic Microwave Background in a Universe Dominated by Cold Dark Matter. Astrophysical Journal, 285, L39-L43. https://doi.org/10.1086/184361

[18] Kleinert, H. (2016) Particle and Quantum Fields. World Scientific Publishing, Hackensack. https://doi.org/10.1142/9915

[19] Bekenstein, J. and Milgrom, M. (1984) Does the Missing Mass Problem Signal the Breakdown of Newtonian Gravity? Astrophysical Journal, 286, 7-14. https://doi.org/10.1086/162570

[20] Milgrom, M. (1983) A Modification of the Newtonian Dynamics-Implications for Galaxies. Astrophysical Journal, 270, 371-389. https://doi.org/10.1086/161131

[21] Bekenstein, J.D. (2009) Relativistic MOND as an Alternative to the Dark Matter Paradigm. Nuclear Physics, A, 827, 555c-560c. https://doi.org/10.1016/j.nuclphysa.2009.05.122

[22] Milgrom, M. (2010) Quasi-Linear Formulation of MOND. Monthly Notices of the Royal Astronomical Society, 403, 886-895. https://doi.org/10.1111/j.1365-2966.2009.16184.x

[23] Bekenstein, J.D. (2005) Erratum: Relativistic Gravitation Theory for the Modified Newtonian Dynamics Paradigm [Physical Review D, 71, Article ID: 069901 (2004)]. Physical Review D, 70, Article ID: 083509. https://doi.org/10.1103/PhysRevD.71.069901

[24] Boran, S., Desai, S., Kahya, E.O. and Woodard, R.P. (2018) GW170817 Falsifies Dark Matter Emulators. Physical Review D, 97, Article ID: 041501. https://doi.org/10.1103/PhysRevD.97.041501

[25] Alcock, C., Allsman, R.A., Alves, D.R., Axelrod, T.S., Becker, A.C., Bennett, D.P., et al. (2000) The MACHO Project: Microlensing Results from 5.7 Years of Large Magellanic Cloud Observations. The Astrophysical Journal, 542, 281-307. https://doi.org/10.1086/309512

[26] Expérience pour la Recherche d'Objets Sombres (n.d.) Presentation of EROS. http://eros.in 2 p3.fr/presentation.html

[27] Wyrzykowski, L., et al. (2011) The OGLE View of Microlensing towards the Magellanic Clouds-IV. OGLE-III SMC Data and Final Conclusions on MACHOs. Monthly Notices of the Royal Astronomical Society, 416, 2949-2961. https://doi.org/10.1111/j.1365-2966.2011.19243.x

[28] Calcino, J., García-Bellido, J. and Davis, T.M. (2018) Updating the MACHO Fraction of the Milky Way Dark Halo with Improved Mass Models. Monthly Notices of the Royal Astronomical Society, 479, 2889-2905. https://doi.org/10.1093/mnras/sty1368

[29] Niikura, H., Takada, M., Yasuda, N., Lupton, R.H., Sumi, T., More, S., et al. (2019) Microlensing Constraints on Primordial Black Holes with Subaru/HSC Andromeda Observations. Nature Astronomy, 3, 524-534. 
https://doi.org/10.1038/s41550-019-0723-1

[30] Jungman, G., Kamionkowski, M. and Griest K. (1996) Supersymmetric Dark Matter. Physics Reports, 267, 195-373. https://doi.org/10.1016/0370-1573(95)00058-5

[31] Schumann, M. (2019) Direct Detection of WIMP Dark Matter: Concepts and Status. Journal of Physics G: Nuclear and Particle Physics, 46, Article ID: 103003. https://doi.org/10.1088/1361-6471/ab2ea5

[32] http://cosmo17.in2p3.fr/talks/plenary/weniger COSMO17.pdf

[33] Gilman, D., Birrer, S., Nierenberg, A., Treu, T., Du, X. and Benson, A. (2019) Warm Dark Matter Chills Out: Constraints on the Halo Mass Function and the Free-Streaming Length of Dark Matter with Eight Quadruple-Image Strong Gravitational Lenses. Monthly Notices of the Royal Astronomical Society, 491, 6077-6101. https://doi.org/10.1093/mnras/stz3480

[34] Almeida, J.S., Trujillo, I. and Plastino, A.R. (2020) The Principle of Maximum Entropy Explains the Cores Observed in the Mass Distribution of Dwarf Galaxies. Astronomy \& Astrophysics, 642, Article No. L14. https://doi.org/10.1051/0004-6361/202039190

[35] Siegel, E. (2020) The Universe Would Be Very Different without Dark Matter. Forbes Editor's Pick.

https://www.forbes.com/sites/startswithabang/2020/02/14/the-universe-would-be-v ery-different-without-dark-matter/?sh=319f7e0637ea-: : :text=Withoutdarkmatter,g alaxieswouldlosealarge, thatformsnewstarsthanmodern galaxie

[36] Lemaître, G. (1927) Un Univers homogène de masse constante et de rayon croissant rendant compte de la vitesse radiale des nébuleuses extra-galactiques. Annales de la Société Scientifique de Bruxelles, A47, 49-59.

[37] Eddington, A.S. (1924) A Comparison of Whitehead's and Einstein's Formulæ. Nature, 113, 192. https://doi.org/10.1038/113192a0

[38] Hubble, E. (1929) A Relation between Distance and Radial Velocity among Extra-Galactic Nebulae. Proceedings of the National Academy of Sciences of the United States of America, 15, 168-173. https://doi.org/10.1073/pnas.15.3.168

[39] Freedman, W.L., Madore, B.F., Hatt, D., Hoyt, T.J., Jang, I.S., Beaton, R.L., et al. (2019) The Carnegie-Chicago Hubble Program. VIII. An Independent Determination of the Hubble Constant Based on the Tip of the Red Giant Branch. The Astrophysical Journal, 882, Article No. 34. https://doi.org/10.3847/1538-4357/ab2f73

[40] The LIGO Scientific Collaboration and the Virgo Collaboration (2019) A Gravitational-Wave Measurement of the Hubble Constant Following the Second Observing Run of Advanced LIGO and Virgo. https://dcc-llo.ligo.org/LIGO-P1900015/public

[41] Young, M. (2019) Tension over Hubble Constant Continues. https://skyandtelescope.org/astronomy-news/tension-continues-hubble-constant/

[42] Lombriser, L. (2020) Consistency of the Local Hubble Constant with the Cosmic Microwave Background. Physics Letters B, 803, Article ID: 135303. https://doi.org/10.1016/j.physletb.2020.135303

[43] Riess, A.G., Filippenko, A.V., Challis, P., Clocchiatti, A., Diercks, A., Garnavich, P.M., et al. (1998) Observational Evidence from Supernovae for an Accelerating Universe and a Cosmological Constant. The Astronomical Journal, 116, 1009-1038. https://doi.org/10.1086/300499

[44] Perlmutter, S., Aldering, G., Goldhaber, G., Knop, R.A., Nugent, P., Castro, P.G., et al. (1999) Measurements of $\Omega$ and $\Lambda$ from 42 High-Redshift Supernovae. The Astrophysical Journal, 517, 565-586. https://doi.org/10.1086/307221 
[45] Matthews, R. (1998) Dirac's Coincidences Sixty Years on. Astronomy \& Geophysics, 39, 6.19-6.20. https://doi.org/10.1093/astrog/39.6.6.19

[46] Dirac, P.A.M. (1937) The Cosmological Constants. Nature, 139, 323. https://doi.org/10.1038/139323a0

[47] Dirac, P.A.M. (1938) A New Basis for Cosmology. Proceedings of the Royal Society $A, 165,199-208$. https://doi.org/10.1098/rspa.1938.0053

[48] Dirac, P.A.M. (1974) Cosmological Models and the Large Numbers Hypothesis. Proc. R. Soc. A, 338, 439-446. https://doi.org/10.1098/rspa.1974.0095

[49] Shao, C.G., Shen, J., Wang, B. and Su, R.K. (2006) Dirac Cosmology and the Acceleration of the Contemporary Universe. Classical and Quantum Gravity, 23, Article No. 3707. https://doi.org/10.1088/0264-9381/23/11/003

[50] Bondi, H. (1957) Negative Mass in General Relativity. Reviews of Modern Physics, 29, 423-428. https://doi.org/10.1103/RevModPhys.29.423

[51] Hossenfelder, S. (2018) Antigravitation. Summary of the 17th International Conference on Supersymmetry and the Unification of Fundamental Interactions. AIP, 1200, 1089-1092.

[52] Mena Marugan, G.A. and Carneiro, S. (2002) Holography and the Large Number Hypothesis. Physical Review D, 65, Article ID: 087303. https://doi.org/10.1103/PhysRevD.65.087303

[53] Ray, S., Mukhopadhyay, U. and Ghosh, P.P. (2007) Large Number Hypothesis: A Review. Unpublished. https://arxiv.org/abs/0705.1836

[54] Unzicker, A. (2009) A Look at the Abandoned Contributions to Cosmology of Dirac, Sciama, and Dicke. Annalen der Physik, 18, 57-70.

https://doi.org/10.1002/andp.200810335

[55] Lehmkuhl, D. (2014) Why Einstein Did Not Believe That General Relativity Geometrizes Gravity. Studies in History and Philosophy of Science Part B: Studies in History and Philosophy of Modern Physics, 46, 316-326.

https://doi.org/10.1016/j.shpsb.2013.08.002

[56] Falk, D. (2016) A Debate Over the Physics of Time. https://www.quantamagazine.org/a-debate-over-the-physics-of-time-20160719/

[57] Minkowski, H. (2012) Space and Time: Minkowski’s Papers on Relativity. Minkowski Institute Press, Montreal.

[58] Einstein, A. (1914) Die formale Grundlage der allgemeinen Relativitätstheorie. Sitzungsberichte der Preußischen Akademie der Wissenschaften, Berlin, 1030-1085.

[59] Poincaré, H. (1903) L'espace et ses trois dimensions. Revue de Métaphysique et de Morale, 11, 407-429.

[60] Pardo, K., Fishbach, M., Holz, D.E. and Spergel, D.N. (2018) Limits on the Number of Spacetime Dimensions from GW170817. Journal of Cosmology and Astroparticle Physics, 2018, Article No. 48. https://doi.org/10.1088/1475-7516/2018/07/048

[61] Tegmark, M. (1997) On the Dimensionality of Spacetime. Classical and Quantum Gravity, 14, L69-L75. https://doi.org/10.1088/0264-9381/14/4/002

[62] Feynman, R.P. (1963) Space-Time. In: Feynman, R., Leighton, R. and Sands, M., Eds., The Feynman Lectures on Physics, Vol. 2, California Institute of Technology, Pasadena, 42. https://www.feynmanlectures.caltech.edu/I 17.html

[63] (2017) Evidence for Dark Matter. https://www.chandra.harvard.edu/xray astro/dark matter/index2.html

[64] Markevitch, M., Gonzalez, A.H., Clowe, D., Vikhlinin, A., Forman, W., Jones, C., et 
al. (2004) Direct Constraints on the Dark Matter Self-Interaction Cross Section from the Merging Galaxy Cluster 1E 0657-56. The Astrophysical Journal, 606, 819. https://doi.org/10.1086/383178

[65] Massey, R., Rhodes, J., Ellis, R.S., Scoville, N., Leauthaud, A., Finoguenov, A., et al. (2007) Dark Matter Maps Reveal Cosmic Scaffolding. Nature, 445, 286-290. https://doi.org/10.1038/nature05497

[66] Wechsler, R.H. and Tinker, J.L. (2018) The Connection between Galaxies and their Dark Matter Halos. Annual Review of Astronomy and Astrophysics, 56, 435-487. https://doi.org/10.1146/annurev-astro-081817-051756

[67] National Aeronautics and Space Administration (2016) Cobe. National Aeronautics and Space Administration, Washington DC. https://science.nasa.gov/missions/cobe

[68] Kogut, A., Lineweaver, C., Smoot, G.F., Bennett, C.L., Banday, A., Boggess, N.W., et al. (1993) Dipole Anisotropy in the COBE Differential Microwave Radiometers First-Year Sky Maps. Astrophysical Journal, 419, 1. https://doi.org/10.1086/173453

[69] Fixsen, D.J., Cheng, E.S., Gales, J.M., Mather, J.C., Shafer, R.A. and Wright, E.L. (1996) The Cosmic Microwave Background Spectrum from the Full COBE FIRAS Data Set. The Astrophysical Journal, 473, 576-587. https://doi.org/10.1086/178173

[70] National Aeronautics and Space Administration (2013) WMAP Produces New Results. National Aeronautics and Space Administration, Washington DC. https://map.gsfc.nasa.gov/news/index.html

[71] Bennett, C.L., Larson, D., Weiland, J.L., Jarosik, N., Hinshaw, G., Odegard, N., et al. (2013) Nine-Year Wilkinson Microwave Anisotropy Probe (WMAP) Observations: Final Maps and Results. The Astrophysical Journal Supplement Series, 208, 20. https://doi.org/10.1088/0067-0049/208/2/20

[72] European Space Agemcy (2018) Planck. http://www.esa.int/Science Exploration/Space Science/Planck

[73] Planck Collaboration (2014) Planck 2013 Results. XVI. Cosmological Parameters. Astronomy \& Astrophysics, 571, Article No. A16.

[74] Wilkins, G.A. (1989) IAU Style Manual. Comm. 5 in IAU Transactions XXB. International Astronomical Union, Paris.

[75] Scott, D. (2019) 29. Cosmic Microwave Background. http://pdg.ge.infn.it/2020/reviews/rpp2020-rev-cosmic-microwave-background.pdf

[76] National Aeronautics and Space Administration (2021) Physical CDM Density, $\Omega_{\mathrm{c}} \mathrm{h}^{2}$. https://lambda.gsfc.nasa.gov/education/graphic history/cdmdensity.cfm

[77] National Aeronautics and Space Administration (2019) New Hubble Constant Measurement Adds to Mystery of Universe's Expansion Rate. https://hubblesite.org/contents/news-releases/2019/news-2019-28

[78] Abbott, T.M.C., Allam, S., Andersen, P., Angus, C., Asorey, J., Avelino, A., et al. (2019) First Cosmology Results Using Type Ia Supernovae from the Dark Energy Survey: Constraints on Cosmological Parameters. The Astrophysical Journal Letters, 872, L30. https://doi.org/10.3847/2041-8213/ab04fa

[79] Tinker, J.L., Leauthaud, A., Bundy, K., et al. (2018) Evolution of the Stellar-to-Dark Matter Relation: Separating Star-Forming and Passive Galaxies from $\mathrm{z}=1$ to 0 . Annual Review of Astronomy and Astrophysics, 1, 56.

[80] Khamehchi, M. A., Hossain, K., Mossman, M.E., Zhang, Y., Busch, T., McNeil Forbes, M., et al. (2017) Negative-Mass Hydrodynamics in a Spin-Orbit-Coupled Bose-Einstein Condensate. Physical Review Letters, 118, Article ID: 155301. 
https://doi.org/10.1103/PhysRevLett.118.155301

[81] Farnes, J.S. (2018) A Unifying Theory of Dark Energy and Dark Matter: Negative Masses and Matter Creation within a Modified $\Lambda$ CDM Framework. Astronomy \& Astrophysics, 620, Article No. A92. https://doi.org/10.1051/0004-6361/201832898

[82] NASA, ESA, Burchett, J. and Elek, O. (2020) Map of the Cosmic Web Generated from Slime Mould Algorithm. https://esahubble.org/images/heic2003a/

[83] Buote, D.A. and Barth, A.J. (2019) The Extremely High Dark Matter Halo Concentration of the Relic Compact Elliptical Galaxy Mrk 1216. The Astrophysical Journal, 877, 91. https://doi.org/10.3847/1538-4357/ab1008

[84] LIGO (2017) GW170814: A Three-Detector Observation of Gravitational Waves from a Binary Black Hole Coalescence. https://ligo.org/science/Publication-GW170814/index.php

[85] Corda, C. (2009) Interferometric Detection of Gravitational Waves: The Definitive Test for General Relativity. International Journal of Modern Physics D, 18, 2275-2282. https://doi.org/10.1142/S0218271809015904

\section{Further Reading}

- Hubble, E.P. (1937) The Observational Approach to Cosmology. Nature, 138, 1001-1002. https://doi.org/10.1038/1381001a0

- Kutner, M. (2003) Astronomy: A Physical Perspective. 2nd Edition, Cambridge University Press, Cambridge. https://doi.org/10.1017/CBO9780511802195

- Liddle, A.R. (2003) An Introduction to Modern Cosmology. 2nd Edition, John Wiley \& Sons Ltd., Chichester.

- Freedman, W.L. and Madore, B.F. (2010) The Hubble Constant. Annual Review of Astronomy and Astrophysics, 48, 673-710.

https://doi.org/10.1146/annurev-astro-082708-101829 